1 Secretaria Estadual de Saúde do Rio de Janeiro (SES-RJ), Superintendência de Atenção Básica - Rio de Janeiro (RJ), Brasil. josiane.medrado@saude. rj.gov.br

2 Fundação Oswaldo Cruz (Fiocruz), Escola Nacional de Saúde Pública Sergio Arouca (Ensp) - Rio de Janeiro (RJ), Brasil. angelacasanova@ensp. fiocruz.br

3 Fundação Oswaldo Cruz (Fiocruz), Escola Nacional de Saúde Pública Sergio Arouca (Ensp) - Rio de Janeiro (RJ), Brasil.

catiaoliveira@ensp.fiocruz.br

\section{Estudo avaliativo do processo de trabalho das Equipes de Atenção Básica a partir do PMAQ-AB}

\author{
Evaluative study of the working process of Primary Care Teams from \\ $P M A Q-A B$
}

Josiane Ribeiro Silva Medrado $\mathbf{1}$, Angela Oliveira Casanova², Cátia Cristina Martins de Oliveira ${ }^{\mathbf{3}}$

RESUMO O estudo objetivou analisar a organização do processo de trabalho das Equipes de Atenção Básica das regiões de saúde do estado do Rio de Janeiro, a partir dos resultados do primeiro ciclo da Avaliação Externa do PMAQ-AB. Foram consideradas para análise quatro subdimensões do módulo II, cotejadas com as características do processo de trabalho da Política Nacional de Atenção Básica. Observou-se que o processo de Avaliação Externa estimulou o planejamento das ações das equipes, contudo ainda possuem entraves tanto para a gestão quanto para as equipes sobre o aproveitamento das informações em saúde, a precariedade de recursos humanos e a institucionalização da avaliação propriamente dita.

PALAVRAS-CHAVE Atenção Primária a Saúde; Avaliação em saúde; Processo de trabalho em saúde.

ABSTRACT The study aimed to analyze the organization of the working process of the Primary Care Teams of the health regions of the state of Rio de Janeiro, from the results of the first $P M A Q-A B$ external evaluation cycle. They were considered for analysis four sub-dimensions of the module II, collated with the characteristics of the working process of the National Policy of Primary Care. It was observed that the external evaluation process stimulated the planning of the action teams, yet that are still obstacles both for management as for the teams on the use of health information, the precariousness of human resources, and the evaluation of institutionalization itself.

KEYWORDS Primary Health Care; Health evaluation; Working process in health. 


\section{Introdução}

A avaliação entendida como base para os demais componentes da gestão (planejamento, execução, financiamento e regulação) pode subsidiar intervenções mais efetivas no contexto da Rede de Atenção à Saúde (RAS). Nesse sentido, a avaliação em saúde deve ser compreendida em termos do seu papel estratégico para subsidiar processos decisórios e no aspecto tático-operacional utilizada para melhorar a capacidade organizacional da Atenção Básica (AB), favorecer a comunicação e a informação e garantir a transparência na gestão.

A Estratégia Saúde da Família (ESF), modelo predominante de $\mathrm{AB}$ (ou atenção primária) no País, apresenta inegável aumento de cobertura populacional ao longo dos últimos 20 anos e esforços para a sua qualificação vêm sendo realizados com a perspectiva de garantia do acesso e da qualidade na prestação do cuidado. $\mathrm{O}$ Programa Nacional de Melhoria do Acesso e da Qualidade da Atenção Básica (PMAQAB) é uma das propostas apresentadas pelo Ministério da Saúde com a finalidade de ampliar a capacidade de gestão, visando a análise do acesso e da qualidade das ações de saúde no âmbito da $\mathrm{AB}$ nas três esferas de governo. Em seu âmbito foi realizada a Avaliação Externa, que contou com a participação de instituições de ensino e pesquisa na organização e execução do trabalho de campo. Com efeito, fomenta a institucionalização da cultura de avaliação pautada no fortalecimento de iniciativas que reconheçam a qualidade dos serviços de saúde ofertados, visando estimular a ampliação do acesso nos variados contextos brasileiros.

Nesse sentido, o presente artigo tem como propósito provocar a reflexão acerca do uso da avaliação na organização do processo de trabalho das Equipes de Atenção Básica (EqAB) das regiões de saúde do estado do Rio de Janeiro, a partir da análise dos resultados do primeiro ciclo da Avaliação Externa do
PMAQ-AB, correlacionando dimensões nele utilizadas para avaliação do planejamento e organização do processo de trabalho com aquelas previstas na Política Nacional de Atenção Básica (PNAB-2012).

\section{Material e métodos}

Trata-se de um estudo descritivo de abordagem quantitativa, utilizando-se como unidade de análise as regiões de saúde do estado Rio de Janeiro, a saber: Baía da Ilha Grande, Baixada Litorânea, CentroSul, Médio-Paraíba, Metropolitana I, Metropolitana II, Noroeste, Norte e Serrana.

Como fonte de dados, utilizou-se os resultados oriundos do primeiro ciclo da Avaliação Externa, realizado com as EqAB do estado do Rio de Janeiro que fizeram adesão ao PMAQ-AB no ano de 2012. Para tanto, foi utilizado o Módulo II: Entrevista com Profissional da Equipe de Atenção Básica e Verificação de Documentos na Unidade de Saúde, tendo como recorte a Dimensão IV: Acesso e Qualidade da Atenção e Organização do processo de trabalho, cujas subdimensões de análise foram: 1) Planejamento das ações da Equipe de Atenção Básica; 2) Reunião da Equipe de Atenção Básica; 3) Acolhimento à Demanda Espontânea; 4) Organização da Agenda da Equipe de Atenção Básica. Vale ressaltar que não foram utilizadas para análise todas as variáveis das subdimensões citadas. As características do processo de trabalho das EqAB contidas na Política Nacional de Atenção Básica foram priorizadas de acordo com as subdimensões do PMAQ-AB (quadro 1).

Para a análise dos dados, foi utilizado o software SPSS (Statistical Package for the Social Sciences). A aprovação para realização deste processo foi obtida junto ao Comitê de Ética em Pesquisa da Escola Nacional de Saúde Pública/Fiocruz, mediante protocolo $\mathrm{n}^{\mathrm{0}} 32012 / 2012$. 


\section{Resultados e discussão}

No estado do Rio de Janeiro, dos 92 municípios, 77 (83,7\%) aderiram ao PMAQ-AB no primeiro ciclo, com um total de $1.075 \mathrm{EqAB}$. Foram avaliadas 1.047 equipes, sendo, na região Baía da Ilha Grande, 24 equipes (2,3\%); Baixada litorânea, 55 equipes ( $5,3 \%)$; CentroSul, 84 equipes (8,0\%); Médio-Paraíba,
145 equipes (13,8\%); Metropolitana I, 414 equipes (39,5\%); Metropolitana II, 187 equipes (17,8\%); Noroeste, 35 equipes $(3,4 \%)$; Norte, 34 equipes $(3,3 \%)$ e Serrana, 69 equipes (6,5\%). Foram certificadas 1.012 EqAB, o que corresponde a $96,7 \%$ do total de equipes avaliadas no estado. Os resultados serão apresentados abaixo segundo as dimensões definidas no estudo.

Quadro 1. Componentes do processo de trabalho das Equipes de Atenção Básica de acordo com a PNAB e dimensões da Avaliação Externa do PMAQ-AB

\begin{tabular}{|c|c|}
\hline $\begin{array}{l}\text { Política Nacional de Atenção Básica - PNAB } \\
\text { Características do processo de trabalho das Equipes } \\
\text { de Atenção Básica }\end{array}$ & $\begin{array}{l}\text { Módulo II: Dimensão IV - Acesso e Qualidade da Atenção e } \\
\text { Organização do Processo de Trabalho } \\
\text { Subdimensões/Variáveis do questionário do PMAQ-AB }\end{array}$ \\
\hline $\begin{array}{l}\text { 1. Realização do acolhimento com escuta qualificada, } \\
\text { classificação de risco, avaliação de necessidade de } \\
\text { saúde e análise de vulnerabilidade }\end{array}$ & $\begin{array}{l}\text { II. } 15 \text { - Acolhimento à Demanda Espontânea } \\
\text { II. } 16 \text { - Organização da Agenda }\end{array}$ \\
\hline \multirow{2}{*}{ 2. Planejamento e organização do trabalho } & $\begin{array}{l}\text { II. } 9 \text { - Planejamento da Equipe de Atenção Básica e ações da } \\
\text { gestão para a organização do processo de trabalho da equipe }\end{array}$ \\
\hline & II. 11 - Reunião da Equipe de Atenção Básica \\
\hline 3. Monitoramento e avaliação das ações das equipes & $\begin{array}{l}\text { II. } 9 \text { - Planejamento da Equipe de Atenção Básica e ações da } \\
\text { gestão para a organização do processo de trabalho da equipe }\end{array}$ \\
\hline
\end{tabular}

Fonte: Banco de dados PMAQ-AB, primeiro ciclo 2012

\section{Acolhimento, avaliação de risco e vulnerabilidade}

Das EqAB avaliadas no estado do Rio de Janeiro, 93,1\% possuem o acolhimento implantado nas unidades básicas de saúde, sendo que $58,7 \%$ possuem protocolos com definição de diretrizes terapêuticas à demanda de urgência/emergência.

Segundo o Caderno de Atenção Básica, n. 28 'Acolhimento à demanda espontânea' a utilização de tais protocolos e de suas respectivas escalas, reflete na qualidade do acesso destes serviços. Esses protocolos tentam fornecer não um diagnóstico, mas uma prioridade, o que permite impactar sobre a história das doenças agudas graves e potencialmente fatais, que, se não atendidas como prioridade, podem levar à morte (BRASIL, 2012B).

Nesse contexto, o acolhimento é considerado como um mecanismo de ampliação e facilitação do acesso, na medida em que pressupõe tanto o acompanhamento de grupos populacionais portadores de agravos mais prevalentes ou a partir dos ciclos de vida quanto de pessoas que apresentam necessidades de saúde que não estão contempladas nesses critérios. Para isso, o acolhimento deve ser visto como um dispositivo de (re)organização do processo de trabalho da equipe, provocando mudanças tanto nos 
modus operandi das equipes quanto nos modos de cuidar.

No estado, 91,9\% das EqAB afirmam realizar avaliação de risco e vulnerabilidade no acolhimento dos usuários. Todas as regiões apresentaram percentual igual ou superior a $80 \%$. Entretanto, a capacitação dos profissionais para a realização dessa atividade (tabela 1) foi de $65 \%$ no total. Entre as regiões, observa-se uma discrepância quando se compara os percentuais relativos ao desenvolvimento dessa ação e a capacitação das equipes para esse fim.

As regiões que tiveram resultados aproximados em relação à realização da avaliação de risco e capacitação (tabela 1), respectivamente, foram a Metropolitana II (95,7\% e $79 \%$ ) e a Metropolitana I (92,9\% e $74 \%$ ), o que pode ser compreendido, tendo em vista a maior proximidade dos municípios dessas regiões com leque mais amplo de instituições formadoras. As regiões com baixos percentuais de capacitação de equipes para avaliação de risco e vulnerabilidade são: Noroeste (22,7\%), Baía da Ilha Grande (26,1\%) e Centro-Sul $(44,4 \%)$, o que põe em questão como garantir um acolhimento de qualidade sem que os profissionais estejam capacitados e/ou, por outro lado, se há alguma incongruência na informação colhida.

Tabela 1. Acolhimento realizado pelas Equipes de Atenção Básica

\begin{tabular}{|c|c|c|c|c|c|c|c|c|c|c|}
\hline Regiões de Saúde & $\begin{array}{c}\text { Baía } \\
\text { de Ilha } \\
\text { Grande }\end{array}$ & $\begin{array}{l}\text { Baixada } \\
\text { Litorânea }\end{array}$ & $\begin{array}{l}\text { Centro- } \\
\text { Sul }\end{array}$ & $\begin{array}{l}\text { Médio } \\
\text { Paraíba }\end{array}$ & Metropolitana I & Metropolitana II & Norte & Noroeste & Serrana & Total \\
\hline $\begin{array}{l}\text { Total de equipes } \\
\text { certificadas }\end{array}$ & $\begin{array}{c}24 \\
(2,3 \%)\end{array}$ & $\begin{array}{c}55 \\
(5,3 \%)\end{array}$ & $\begin{array}{c}84 \\
(8,0 \%)\end{array}$ & $\begin{array}{c}145 \\
(13,8 \%)\end{array}$ & $414(39,5 \%)$ & $187(17,8 \%)$ & $\begin{array}{c}34 \\
(3,3 \%)\end{array}$ & $\begin{array}{c}35 \\
(3,4 \%)\end{array}$ & $\begin{array}{c}69 \\
(6,5 \%)\end{array}$ & $\begin{array}{c}1012 \\
(100 \%)\end{array}$ \\
\hline \multicolumn{11}{|l|}{ Acolhimento } \\
\hline $\begin{array}{l}\text { Os profissionais da } \\
\text { equipe que fazem } \\
\text { o acolhimento } \\
\text { foram capacitados } \\
\text { para avaliação } \\
\text { e classificação } \\
\text { de risco e } \\
\text { vulnerabilidade dos } \\
\text { usuários? }\end{array}$ & $26,1 \%$ & $51 \%$ & $44,4 \%$ & $57,8 \%$ & $74 \%$ & $79 \%$ & $63,6 \%$ & $22,7 \%$ & $55,7 \%$ & $65 \%$ \\
\hline $\begin{array}{l}\text { A equipe realiza } \\
\text { avaliação de risco e } \\
\text { vulnerabilidade no } \\
\text { acolhimento dos } \\
\text { usuários? }\end{array}$ & $91,7 \%$ & $94,1 \%$ & $87,8 \%$ & $92,6 \%$ & $92,9 \%$ & $95,7 \%$ & $90,9 \%$ & $78,3 \%$ & $82,5 \%$ & $91,9 \%$ \\
\hline \multicolumn{11}{|l|}{$\begin{array}{l}\text { Ações decorrentes } \\
\text { do acolhimento }\end{array}$} \\
\hline $\begin{array}{l}\text { Marcação na } \\
\text { agenda do médico }\end{array}$ & $87,5 \%$ & $92,3 \%$ & $96,4 \%$ & $100 \%$ & $97,7 \%$ & $97,5 \%$ & $87 \%$ & $97 \%$ & $100 \%$ & $97,2 \%$ \\
\hline $\begin{array}{l}\text { Marcação na } \\
\text { agenda do } \\
\text { enfermeiro }\end{array}$ & $0 \%$ & $3,8 \%$ & $0 \%$ & $0 \%$ & $1,8 \%$ & $6 \%$ & $0 \%$ & $0 \%$ & $0 \%$ & $1 \%$ \\
\hline $\begin{array}{l}\text { Marcação na } \\
\text { agenda do dentista }\end{array}$ & $70,8 \%$ & $65,4 \%$ & $81,9 \%$ & $71,3 \%$ & $70,4 \%$ & $30,7 \%$ & $69,6 \%$ & $60,6 \%$ & $65,6 \%$ & $63,9 \%$ \\
\hline $\begin{array}{l}\text { Marcação na } \\
\text { agenda de outro } \\
\text { profissional }\end{array}$ & $87,5 \%$ & $34,6 \%$ & $65,1 \%$ & $88,2 \%$ & $48,9 \%$ & $70,6 \%$ & $65,2 \%$ & $75,8 \%$ & $48,4 \%$ & $60,8 \%$ \\
\hline
\end{tabular}




\begin{tabular}{lcccccccccc}
\hline $\begin{array}{l}\text { Tabela 1. (cont.) } \\
\text { Orientação }\end{array}$ & $100 \%$ & $75 \%$ & $97,6 \%$ & $97,8 \%$ & $92,9 \%$ & $92,6 \%$ & $95,7 \%$ & $100 \%$ & $93,8 \%$ & $93,5 \%$ \\
\hline $\begin{array}{l}\text { Solicitação de } \\
\text { exames }\end{array}$ & $87,5 \%$ & $63,5 \%$ & $78,3 \%$ & $76,5 \%$ & $83,5 \%$ & $70,6 \%$ & $65,2 \%$ & $72,7 \%$ & $78,1 \%$ & $77,8 \%$ \\
$\begin{array}{l}\text { Medidas } \\
\text { terapêuticas }\end{array}$ & $87,5 \%$ & $75 \%$ & $88 \%$ & $80,9 \%$ & $85,1 \%$ & $83,4 \%$ & $73,9 \%$ & $93,9 \%$ & $84,4 \%$ & $84 \%$ \\
$\begin{array}{l}\text { Prescrição de } \\
\text { alguma medicação }\end{array}$ & $79,2 \%$ & $67,3 \%$ & $60,2 \%$ & $73,5 \%$ & $78,7 \%$ & $70,6 \%$ & $65,2 \%$ & $78,8 \%$ & $71,9 \%$ & $73,7 \%$ \\
\hline
\end{tabular}

Fonte: Banco de dados PMAQ-AB, primeiro ciclo 2012.

Entre as regiões do estado do Rio de Janeiro, as principais ações oriundas a partir do acolhimento (tabela 1) são: a marcação na agenda do médico (97,2\%), seguida de orientação $(93,5 \%)$ e, em terceiro, medidas terapêuticas (84\%). Nota-se que mesmo sendo a marcação do médico a primeira ação decorrente do acolhimento, tem-se, em seguida, a orientação sem marcação de consulta e as medidas terapêuticas, que não envolvem a prescrição de medicação como ações consequentes do acolhimento. Isso denota a possibilidade de estar ocorrendo uma mudança de paradigma nas equipes em relação às formas de lidar com o cuidado, modificando a lógica médico-centrada e dependente de procedimentos.

A organização da agenda de uma equipe é fundamental na logística da rotina da equipe no que tange ao atendimento das demandas espontânea e programada, bem como para suscitar a conscientização da importância do papel de cada profissional na resolução de cada caso. Do total das EqAB, $84,9 \%$ pactuam uma agenda semanal de atividades com o conjunto de profissionais que integram a equipe.

Quanto ao desenvolvimento de ações para grupos de risco e prioritários, 94,7\% afirmaram que realizam programação na agenda de consultas e ações para esses usuários.

A organização da agenda para a realização de Visitas Domiciliares (VD) é realizada por $93,7 \%$ do total das equipes das regiões de saúde, o que parece refletir a prioridade dada na realização dessa atividade em consonância com o preconizado na PNAB (BRASIL, 2012A), no qual a VD constitui um importante veículo de operacionalização de parte de um programa ou de uma política de assistência à saúde, possibilitando a concretização da longitudinalidade, integralidade, acessibilidade e a interação entre o profissional e o usuário/família (PEREIRA ET AL., 2004).

Certamente, a VD é uma importante estratégia de intervenção, uma vez que a ida do profissional de saúde ao domicílio, muitas vezes, representa uma nova forma de atuação: perde-se a proteção das paredes do consultório, obrigando-o a entrar em contato com as singularidades da família, suas angústias e subjetividades, conhecer o território e sua dinâmica e, ao mesmo tempo, maior compreensão dos processos de saúde e doença e suas formas de cuidado (REIS ET AL., 2007).

Quanto às ações educativas, do total das equipes das regiões de saúde, $82,5 \%$ afirmaram realizar atividade comunitária ou de grupo, com $88,1 \%$ possuindo comprovação documental. Os percentuais inferiores a $80 \%$ encontrados em cinco regiões do estado revelam que as atividades de promoção da saúde não têm sido valorizadas conforme o esperado, considerando-se a mudança do modelo de atenção preconizada pela ESF. Como afirmam Albuquerque e Stotz (2004, P. 264),

no dia a dia dos serviços de saúde, porém, pouca ou nenhuma importância é dada às ações educativas. Trabalhos em grupo são muitas vezes marginalizados, os profissionais envolvidos são desacreditados e desestimulados, a 
infraestrutura necessária é escassa e de difícil acesso aos profissionais.

\section{Planejamento e organização do pro- cesso de trabalho}

Quanto às atividades de planejamento, no total das regiões, mais de $92 \%$ das equipes afirmaram realizá-las. Sob esse aspecto, o levantamento de problemas e a definição de prioridades têm sido realizados por $95,4 \%$ e $96,3 \%$ do total das equipes respectivamente (tabela 2).

Algumas regiões apresentaram percentuais similares quando analisadas a realização das duas atividades, como a Baixada Litorânea com $97,8 \%$, Médio-Paraíba com $97,8 \%$ e Norte com 96,6\% respectivamente. Entretanto, as regiões Centro-Sul, Metropolitana I e Metropolitana II tiveram percentuais maiores na definição de prioridades do que no levantamento de problemas. Como já foi destacado, o PMAQ-AB envolveu um processo prévio de autoavaliação pelas equipes que fizeram adesão ao programa e que implicou exatamente na identificação de problemas e definição de prioridades para o desenho de uma matriz de intervenção. Nesse sentido, tais resultados favoráveis podem refletir esse processo, pois observou-se que, do total das regiões de saúde, 95,8\% das EqAB consideraram os resultados da autoavaliação no planejamento e organização do processo de trabalho da equipe. Esse dado é importante, tendo em vista o incentivo da gestão na autoavaliação das equipes, com o intuito de melhorar a organização e o processo de trabalho delas. De toda forma, 96,2\% das equipes afirmaram ter tido apoio da gestão no planejamento e organização do processo de trabalho. $\mathrm{O}$ apoio às EqAB (tabela 2), seja por um apoiador institucional $(58,3 \%)$ ou profissional de vigilância $(40,5 \%)$, ou outros profissionais da

Tabela 2. Organização e planejamento do processo de trabalho das Equipes de Atenção Básica

\begin{tabular}{|c|c|c|c|c|c|c|c|c|c|c|}
\hline $\begin{array}{l}\text { Regiões de } \\
\text { Saúde }\end{array}$ & $\begin{array}{c}\text { Baia de llha } \\
\text { Grande }\end{array}$ & $\begin{array}{l}\text { Baixada } \\
\text { Litorânea }\end{array}$ & $\begin{array}{l}\text { Centro- } \\
\text { Sul }\end{array}$ & $\begin{array}{l}\text { Médio } \\
\text { Paraíba }\end{array}$ & Metropolitana I & Metropolitana II & Norte & Noroeste & Serrana & Total \\
\hline $\begin{array}{l}\text { Total de equipes } \\
\text { certificadas }\end{array}$ & $24(2,3 \%)$ & $\begin{array}{c}55 \\
(5,3 \%)\end{array}$ & $\begin{array}{c}84 \\
(8,0 \%)\end{array}$ & $\begin{array}{c}145 \\
(13,8 \%)\end{array}$ & $414(39,5 \%)$ & $187(17,8 \%)$ & $\begin{array}{c}34 \\
(3,3 \%)\end{array}$ & $\begin{array}{c}35 \\
(3,4 \%)\end{array}$ & $\begin{array}{c}69 \\
(6,5 \%)\end{array}$ & $\begin{array}{c}1012 \\
(100 \%)\end{array}$ \\
\hline \multicolumn{11}{|l|}{$\begin{array}{l}\text { Organização e } \\
\text { Planejamento }\end{array}$} \\
\hline $\begin{array}{l}\text { Nos últimos } 12 \\
\text { meses, a equipe } \\
\text { realizou alguma } \\
\text { atividade de } \\
\text { planejamento de } \\
\text { suas ações? }\end{array}$ & $87,5 \%$ & $83,3 \%$ & $93,0 \%$ & $96,5 \%$ & $94,6 \%$ & $94,1 \%$ & $85,3 \%$ & $80,0 \%$ & $86,8 \%$ & $92,6 \%$ \\
\hline $\begin{array}{l}\text { Foi realizado } \\
\text { processo de } \\
\text { levantamento de } \\
\text { problemas? }\end{array}$ & $95,2 \%$ & $97,8 \%$ & $92,4 \%$ & $97,8 \%$ & $95,6 \%$ & $93,7 \%$ & $96,6 \%$ & $92,9 \%$ & $96,5 \%$ & $95,4 \%$ \\
\hline $\begin{array}{l}\text { Foram elencadas } \\
\text { prioridades? }\end{array}$ & $90,5 \%$ & $97,8 \%$ & $93,7 \%$ & $97,8 \%$ & $96,9 \%$ & $96,6 \%$ & $96,6 \%$ & $88,9 \%$ & $96,4 \%$ & $96,3 \%$ \\
\hline $\begin{array}{l}\text { Contribuição da } \\
\text { autoavaliação } \\
\text { no processo de } \\
\text { trabalho }\end{array}$ & $94,40 \%$ & $97,90 \%$ & $98,60 \%$ & $99,30 \%$ & $95,00 \%$ & $94,60 \%$ & $87,50 \%$ & $90,90 \%$ & $96,50 \%$ & $95,80 \%$ \\
\hline
\end{tabular}




\begin{tabular}{|c|c|c|c|c|c|c|c|c|c|c|}
\hline \multicolumn{11}{|l|}{$\begin{array}{l}\text { Apoio } \\
\text { institucional }\end{array}$} \\
\hline $\begin{array}{l}\text { A equipe recebe } \\
\text { apoio para o } \\
\text { planejamento } \\
\text { e organização } \\
\text { do processo de } \\
\text { trabalho? }\end{array}$ & $57,1 \%$ & $77,3 \%$ & $92,5 \%$ & $93,4 \%$ & $88,9 \%$ & $90,9 \%$ & $58,6 \%$ & $71,4 \%$ & $83,1 \%$ & $87,2 \%$ \\
\hline $\begin{array}{l}\text { Apoiador } \\
\text { institucional }\end{array}$ & $41,7 \%$ & $47,1 \%$ & $24,3 \%$ & $59,8 \%$ & $67,3 \%$ & $64,2 \%$ & $23,5 \%$ & $50,0 \%$ & $51,0 \%$ & $58,3 \%$ \\
\hline $\begin{array}{l}\text { Profissionais da } \\
\text { vigilância em } \\
\text { saúde }\end{array}$ & $33,3 \%$ & $14,7 \%$ & $25,7 \%$ & $40,9 \%$ & $51,2 \%$ & $37,7 \%$ & $17,6 \%$ & $50,0 \%$ & $20,4 \%$ & $40,5 \%$ \\
\hline $\begin{array}{l}\text { Outros } \\
\text { profissionais da } \\
\text { gestão }\end{array}$ & $75,0 \%$ & $52,9 \%$ & $86,5 \%$ & $78,0 \%$ & $69,9 \%$ & $66,0 \%$ & $94,1 \%$ & $75,0 \%$ & $67,3 \%$ & $71,7 \%$ \\
\hline Outro(s) & $0,0 \%$ & $8,8 \%$ & $14,9 \%$ & $14,2 \%$ & $18,1 \%$ & $8,8 \%$ & $17,6 \%$ & $5,0 \%$ & $16,3 \%$ & $14,4 \%$ \\
\hline \multicolumn{11}{|l|}{$\begin{array}{l}\text { Reuniões de } \\
\text { equipe }\end{array}$} \\
\hline $\begin{array}{l}\text { Organização } \\
\text { do processo de } \\
\text { trabalho e do } \\
\text { serviço }\end{array}$ & $100 \%$ & $90,7 \%$ & $98,8 \%$ & $99,3 \%$ & $99,3 \%$ & $98,4 \%$ & $100 \%$ & $97,1 \%$ & $97,1 \%$ & $98,5 \%$ \\
\hline $\begin{array}{l}\text { Discussão de } \\
\text { casos pela } \\
\text { equipe }\end{array}$ & $95,8 \%$ & $81,5 \%$ & $92,9 \%$ & $93,7 \%$ & $97,3 \%$ & $83 \%$ & $85,7 \%$ & $97,1 \%$ & $92,8 \%$ & $92,4 \%$ \\
\hline $\begin{array}{l}\text { Qualificação } \\
\text { clínica com } \\
\text { participação de } \\
\text { equipe de apoio } \\
\text { matricial }\end{array}$ & $20,8 \%$ & $27,8 \%$ & $61,2 \%$ & $69,2 \%$ & $69,3 \%$ & $52,2 \%$ & $31,4 \%$ & $64,7 \%$ & $30,4 \%$ & $58,3 \%$ \\
\hline $\begin{array}{l}\text { Construção } \\
\text { / discussão } \\
\text { de Projeto } \\
\text { Terapêutico } \\
\text { Singular (PTS) }\end{array}$ & $62,5 \%$ & $42,6 \%$ & $57,6 \%$ & $64,3 \%$ & $74,9 \%$ & $50 \%$ & $57,1 \%$ & $58,8 \%$ & $49,3 \%$ & $62,9 \%$ \\
\hline $\begin{array}{l}\text { Planejamento } \\
\text { das ações da } \\
\text { equipe }\end{array}$ & $83,3 \%$ & $94,4 \%$ & $96,5 \%$ & $97,9 \%$ & $98,5 \%$ & $96,2 \%$ & $94,3 \%$ & $100 \%$ & $98,6 \%$ & $97,2 \%$ \\
\hline $\begin{array}{l}\text { Monitoramento } \\
\text { e análise dos } \\
\text { indicadores e } \\
\text { informações de } \\
\text { saúde }\end{array}$ & $66,7 \%$ & $66,7 \%$ & $87,1 \%$ & $92,3 \%$ & $87,8 \%$ & $83 \%$ & $62,9 \%$ & $70,6 \%$ & $79,7 \%$ & $84 \%$ \\
\hline Outros & $4,2 \%$ & $1,9 \%$ & $9,4 \%$ & $12,6 \%$ & $9,7 \%$ & $4,4 \%$ & $2,9 \%$ & $5,9 \%$ & $1,4 \%$ & $7,7 \%$ \\
\hline
\end{tabular}

Fonte: Banco de Dados PMAQ-AB, primeiro ciclo 2012.

gestão $(71,7 \%)$ realizando a função de apoiador institucional, é considerado importante dispositivo para qualificar o planejamento e a organização do processo de trabalho.
A categoria com menor percentual na função de apoio em todas as regiões de saúde refere-se aos profissionais da vigilância em saúde. Isso pode refletir a ausência da integração entre esses profissionais e a EqAB, 
mesmo havendo espaços instituídos para que ela ocorra - reunião de equipe, VD ou mesmo alguma atividade de promoção e prevenção. Quanto ao apoiador institucional, os maiores percentuais foram encontrados nas regiões Metropolitanas I e II (67,3\% e 64,2\% respectivamente). Os baixos percentuais nas demais regiões apontam para o fato dessa estratégia ainda não ter sido incorporada de maneira correta, especialmente no interior do estado, o que pode estar relacionada com a capacidade institucional dos municípios em conter em seu quadro profissionais qualificados para o exercício dessa função.

Conforme aponta Campos (2003), o apoio institucional é uma estratégia metodológica para o enfrentamento dos inúmeros desafios que o trabalho no campo da saúde coloca. A Política Nacional de Humanização indica que a função do apoiador institucional é de contribuir para a gestão e organização de processos de trabalho, na construção de espaços coletivos, nos quais os grupos analisam, definem tarefas e elaboram projetos de intervenção.

Outra estratégia potente para avaliação e organização do processo de trabalho é a reunião de equipe, que favorece a reflexão sobre o andamento das ações e atividades e do rumo a se tomar. Nesse caso, $99 \%$ das $\mathrm{EqAB}$ afirmaram realizá-la, sendo 59,5\% das EqAB com periodicidade semanal, $18 \%$ quinzenal e $13,9 \%$ mensal. Compreende-se que a reunião de equipe é utilizada como um espaço para planejar, organizar e discutir suas ações e seus problemas. Segundo a Política Nacional de Humanização (BRASIL, 2006, P. 54),

a reunião de equipe não é um espaço apenas para que uma pessoa da equipe distribua tarefas às outras. É um espaço de diálogo e é preciso que haja um clima em que todos tenham direito à voz e à opinião.

Nas reuniões de equipe, o planejamento e programação das atividades das EqAB levam em consideração a construção de agenda para organização do processo de trabalho (tabela 3), cujo percentual total é de $94,5 \%$, seguido do uso das informações do Sistema de Informação da Atenção Básica (Siab), com 91,2\%, e, em terceiro lugar, das informações locais, com 88,7\% (diagnóstico da área). Nas regiões de saúde, destacam-se as regiões Médio-Paraíba, Metropolitana I e Metropolitana II, cuja

Tabela 3. Planejamento das atividades das Equipes de Atenção Básica

\begin{tabular}{|c|c|c|c|c|c|c|c|c|c|c|}
\hline Regiões de Saúde & $\begin{array}{c}\text { Baía } \\
\text { de llha } \\
\text { Grande }\end{array}$ & $\begin{array}{l}\text { Baixada } \\
\text { Litorânea }\end{array}$ & $\begin{array}{l}\text { Centro- } \\
\text { Sul }\end{array}$ & $\begin{array}{l}\text { Médio } \\
\text { Paraíba }\end{array}$ & Metropolitana I & Metropolitana II & Norte & Noroeste & Serrana & Total \\
\hline $\begin{array}{l}\text { Total de equipes } \\
\text { certificadas }\end{array}$ & $\begin{array}{c}24 \\
(2,3 \%)\end{array}$ & $\begin{array}{c}55 \\
(5,3 \%)\end{array}$ & $\begin{array}{c}84 \\
(8,0 \%)\end{array}$ & $\begin{array}{c}145 \\
(13,8 \%)\end{array}$ & $414(39,5 \%)$ & $187(17,8 \%)$ & $\begin{array}{c}34 \\
(3,3 \%)\end{array}$ & $\begin{array}{c}35 \\
(3,4 \%)\end{array}$ & $\begin{array}{c}69 \\
(6,5 \%)\end{array}$ & 1012 \\
\hline $\begin{array}{l}\text { Construção de } \\
\text { uma agenda de } \\
\text { trabalho }\end{array}$ & $79,20 \%$ & $90,70 \%$ & $94,10 \%$ & $97,90 \%$ & $97,60 \%$ & $92,30 \%$ & $80,00 \%$ & $100,00 \%$ & $88,40 \%$ & $94,50 \%$ \\
\hline $\begin{array}{l}\text { As metas } \\
\text { pactuadas pelo } \\
\text { município }\end{array}$ & $66,70 \%$ & $59,30 \%$ & $88,20 \%$ & $88,10 \%$ & $91,20 \%$ & $87,40 \%$ & $68,60 \%$ & $82,40 \%$ & $78,30 \%$ & $85,70 \%$ \\
\hline $\begin{array}{l}\text { As informações } \\
\text { do Siab }\end{array}$ & $87,50 \%$ & $92,60 \%$ & $96,50 \%$ & $97,90 \%$ & $90,00 \%$ & $92,90 \%$ & $85,70 \%$ & $94,10 \%$ & $75,40 \%$ & $91,20 \%$ \\
\hline $\begin{array}{l}\text { As informações } \\
\text { locais }\end{array}$ & $87,50 \%$ & $72,20 \%$ & $85,90 \%$ & $93,70 \%$ & $93,40 \%$ & $80,20 \%$ & $85,70 \%$ & $91,20 \%$ & $89,90 \%$ & $88,70 \%$ \\
\hline $\begin{array}{l}\text { As questões } \\
\text { relacionadas } \\
\text { a riscos e } \\
\text { vulnerabilidades }\end{array}$ & $83,30 \%$ & $63,00 \%$ & $87,10 \%$ & $88,80 \%$ & $91,70 \%$ & $75,80 \%$ & $71,40 \%$ & $79,40 \%$ & $81,20 \%$ & $84,70 \%$ \\
\hline
\end{tabular}


Tabela 3. (cont.)

\begin{tabular}{|c|c|c|c|c|c|c|c|c|c|c|}
\hline $\begin{array}{l}\text { As questões } \\
\text { ambientais do } \\
\text { território }\end{array}$ & $70,80 \%$ & $46,30 \%$ & $54,10 \%$ & $69,90 \%$ & $77,80 \%$ & $59,90 \%$ & $60,00 \%$ & $76,50 \%$ & $69,60 \%$ & $68,60 \%$ \\
\hline $\begin{array}{l}\text { Os desafios } \\
\text { apontados } \\
\text { a partir da } \\
\text { autoavaliação }\end{array}$ & $66,70 \%$ & $61,10 \%$ & $80,00 \%$ & $87,40 \%$ & $79,30 \%$ & $79,10 \%$ & $54,30 \%$ & $58,80 \%$ & $81,20 \%$ & $77,80 \%$ \\
\hline
\end{tabular}

Fonte: Banco de Dados PMAQ-AB, primeiro ciclo 2012.

distribuição percentual é bastante próxima, denotando uma igual prioridade entre as atividades elencadas. A região da Baixada Litorânea é a que possui os menores percentuais em relação ao planejamento das atividades.

Foram altos os percentuais encontrados nas regiões quando se questionou as equipes acerca do monitoramento e análise dos indicadores e informações de saúde, excetuando-se as regiões Baía da Ilha Grande (58,3\%), seguida da Noroeste $(60 \%)$ e da Serrana (71,6\%).

\section{Discussão}

O processo de institucionalização da avaliação na $\mathrm{AB}$ tem contribuído para o exercício de uma prática reflexiva e pedagógica, no que tange ao apoio à gestão para a tomada de decisão no âmbito do SUS. Nesse contexto, reconhecer as conexões intrínsecas entre planejamento e avaliação como norteadoras do processo de trabalho tem sido entendida como uma forma de dinamizar e romper com a tradição do planejamento e da avaliação como práticas fragmentadas e burocratizadas (MEDINA; AQUINO; CARVALHO, 2000; FARIA ET AL., 2009). No estudo realizado, verificou-se que, de maneira geral, as regiões de saúde estão, de certa forma, planejando e organizando seu processo de trabalho. Nas regiões consideradas do interior do estado do Rio de Janeiro, como, por exemplo, Baía da Ilha Grande, Baixada Litorânea, Norte e Noroeste, pode-se perceber em algumas dimensões analisadas os baixos percentuais. Isso pode se dever à limitação, tanto da gestão quanto das EqAB desses municípios, em trabalhar em uma lógica voltada para o planejamento, monitoramento e avaliação das ações como elementos intrínsecos e contínuo do processo de trabalho, deixando de ser algo impositivo ou verticalizado com a simples finalidade de se cumprir metas.

Contudo, como condição para a Avaliação Externa, $100 \%$ das equipes das regiões passaram pelo processo da autoavaliação, ainda que o método (instrumento) utilizado tenha sido diferenciado em virtude da possibilidade de escolha da gestão. A adesão das equipes e da gestão a esse processo aponta para a capacidade de incitar essa proposta à institucionalização da avaliação na AB. Entretanto, supõe-se também a questão de uma 'indução' por parte do PMAQ-AB no que tange aos incentivos financeiros envolvidos com a realização da etapa de autoavaliação.

Apesar dos bons resultados, na prática, ainda assim, conforme sinaliza Kawata et al. (2009, P. 317),

há incoerência entre o que a EqAB estabelece como modelo de gestão das equipes, tomando-o como participativo, e o que de fato ocorre, seguindo o modelo centralizado de gestão e com ações de planejamento e monitoramento incipientes.

No que concerne ao apoio da gestão para o processo de trabalho das equipes, percebe-se que as regiões o recebem mesmo que de forma diferenciada, sendo esse apoio dado, em sua maioria, por profissionais da gestão, por apoiador institucional - mais frequente nos municípios de grande porte - ou profissionais da vigilância em saúde. Entretanto, não se sabe de que forma se dá esse apoio, principalmente 
ao levar em consideração a alta rotatividade dos profissionais e a capacidade institucional dos municípios, sobretudo nos de menor porte, em ter no seu quadro profissionais qualificados para tal função.

Por fim, é necessário destacar o reconhecimento das ações de avaliação como instrumento ao processo decisório e de formação dos sujeitos envolvidos, e não as afirmar como mero mecanismo externo.

\section{Considerações finais}

O PMAQ-AB se insere e se relaciona com o conjunto de estratégias prioritárias definidas pela Política Nacional de Atenção Básica e com outros movimentos mais amplos de reorientação do modo como se organiza o SUS, tais como a instituição de redes de atenção e o Programa de Avaliação para a Qualificação do SUS. Nesse âmbito, o PMAQ-AB parece ser uma ferramenta importante na gestão dos recursos destinados à saúde, auxiliando na utilização dos serviços de saúde e contribuindo com melhorias no acesso e na qualidade, corroborando, assim, a tentativa de melhorar o desempenho dos indicadores de saúde. Entretanto, deve-se ressaltar que a incorporação de tais objetivos no processo de trabalho diário das equipes torna-se importante e necessária para que todo o serviço não fique condicionado a apenas um programa.

Este texto aponta como desafio para as EqAB das regiões de saúde do estado do Rio de Janeiro o melhor aproveitamento das informações em saúde para melhoria da gestão, pois se as informações produzidas em saúde forem avaliadas de forma regular, contínua, esta prática será muito mais valiosa para a gestão em todos os níveis, pois além de promover matéria-prima essencial para a análise da situação de saúde de determinada população, facilitará o monitoramento dos objetivos e das metas, além de estimular a capacidade analítica das equipes, promovendo a possibilidade de planejamentos mais efetivos.

Outrossim, para que a avaliação seja entendida como algo realmente importante, no sentido de institucionalizá-la, é necessária, primeiramente, uma vontade dos gestores da $\mathrm{AB}$ dos municípios de colocá-la em prática, desmistificando a ideia de que a avaliação seja vista como de caráter punitivo e fiscalizatório. Essa percepção é muitas vezes reforçada pelos gestores à medida que há uma cobrança sobre indicadores e metas, sem ao menos ser feito um trabalho paralelo com as equipes para esta melhoria, de maneira a incentivá-los e qualificá-los. Isso remete a pensar a forma verticalizada que o PMAQ-AB foi implantado, no sentido de o Ministério da Saúde propor uma adesão voluntária com ausência de esclarecimentos e prazos ínfimos para a discussão do programa com os gestores municipais, pautado em um discurso normativo. Em segundo lugar, precisa haver uma adesão de todos os envolvidos, desde os 'profissionais da ponta' até a gestão, compreendendo que a avaliação é algo pertinente ao processo de trabalho de todos os profissionais de saúde, fomentando nestes a necessidade de olharem de modo reflexivo para suas práticas e, a partir deste processo, atuarem de forma a aprimorá-las. Contudo, a precariedade dos recursos humanos dos municípios, a alta rotatividade de profissionais, o baixo quantitativo de apoio técnico especializado e a sobrecarga de trabalho denotam uma fragilidade de uma realidade a ser superada. Nesse viés, deve haver, por parte dos gestores, o investimento na formação e capacitação do quadro de profissionais, corroborando o aumento de profissionais qualificados para o exercício de determinadas funções.

Implementar as diretrizes do SUS, respeitando os princípios que nortearam sua criação, garantindo sua viabilidade, é o desafio e, muitas vezes, o impasse vivido. 
Atender a essas demandas exige dos gestores uma postura crítica e reflexiva sobre suas práticas, ressaltando-se a avaliação como instrumento fundamental para o aperfeiçoamento das políticas.

\section{Referências}

\author{
ALBUQUERQUE, P. C.; STOTZ, E. N. A educação \\ popular na atenção básica à saúde no município: em \\ busca da integralidade. Interface (Botucatu), Botucatu, \\ v. 8, n. 15 , p. 259-74, 2004.
}

BRASIL. Ministério da Saúde. Portaria MS/GM n. 2.488, de 21 de outubro de 2011. Aprova a Política Nacional de Atenção Básica, estabelecendo a revisão de diretrizes e normas para a organização da Atenção Básica, para a Estratégia Saúde da Família (ESF) e o Programa de Agentes Comunitários de Saúde (PACS). Diário Oficial [da] União, Brasília, DF, 24 out. 2011. Disponível em: <http://bvsms.saude.gov.br/bvs/saudelegis/gm/2011/prt2488_21_10_2011.html>. Acesso em: 23 nov. 2015.

Ministério da Saúde. Secretaria de Atenção à Saúde. Núcleo Técnico da Política Nacional de Humanização. HumanizaSUS: documento base para gestores e trabalhadores do SUS. Brasília, DF: Ministério da Saúde, 2006. Disponível em: <http:// bvsms.saude.gov.br/bvs/publicacoes/documento_base. pdf $>$. Acesso em: 15 mar. 2014.

Ministério da Saúde. Secretaria de Atenção à Saúde. Departamento de Atenção Básica. Política Nacional de Atenção Básica (PNAB). Brasília, DF: Ministério da Saúde, 2012a.

Ministério da Saúde. Secretaria de Atenção à Saúde. Departamento de Atenção Básica. Acolhimento à demanda espontânea: queixas mais comuns na Atenção Básica. Brasília, DF: Ministério da Saúde, 2012b. (Cadernos de Atenção Básica, 28).
CAMPOS, G. W. S. Reflexões Sobre a Clínica Ampliada em Equipes de Saúde da Família. In: CAMPOS, G. W. Saúde Paidéia. São Paulo: Hucitec, 2003. p. 68-77.

FARIA, H. P. et al. Processo de trabalho em saúde. Belo Horizonte, Nescon: UFMG: Coopmed, 2009.

KAWATA, L. S. et al. O trabalho cotidiano da enfermeira na saúde da família: utilização de ferramentas da gestão. Texto \&t Contexto Enferm, Florianópolis, v. 18, n. 2, p. 313-320, 2009.

MEDINA, M. G.; AQUINO, R.; CARVALHO, A. L. B. Avaliação da atenção básica: construindo novas ferramentas para o SUS. Divulgação em Saúde para Debate, Rio de Janeiro, n. 21, p. 15-28, 2000.

PEREIRA, M. J. B. et al. Assistência domiciliar: instrumento para potencializar processos de trabalho na assistência e na formação. In: BARROS, A. F. R. Observatório de Recursos humanos em saúde no Brasil: estudos e análise. Brasília, DF: Ministério da Saúde, 2004. p. 71-80.

REIS, M. A. S. et al. A organização do processo de trabalho em uma unidade de saúde da família: desafios para a mudança das práticas. Interface (Botucatu), Botucatu, v. 11, n. 23, p. 655-666, 2007.

\footnotetext{
Recebido para publicação em maio de 2015

Versão final em setembro de 2015

Conflito de interesses: inexistente

Suporte financeiro: não houve
} 